\title{
Nonlinear Diffusion Equation with Diffusion Coefficient Directly Proportional to Concentration of Impurities
}

\author{
A.J. JANAVIČIUS ${ }^{a, *}$ AND A. PoŠKUs ${ }^{b}$ \\ ${ }^{a}$ Department of Physics and Mathematics, Šiauliai University \\ Višinskio 25, Šiauliai, 76351, Lithuania \\ ${ }^{b}$ Faculty of Physics, Vilnius University, Saulètekio 9, 10222 Vilnius, Lithuania
}

(Received October 27, 2004)

\begin{abstract}
We analyzed a diffusion model based on the assumption that the sufficient condition for the mass flux at point $x+L$ to be different from zero is a nonzero value of the impurity gradient and of impurity concentration at point $x$. In our model, the length of the jump of diffusing particles from one equilibrium position to another has a defined value $L$. By describing variation of impurity concentration with time when the frequency of the jumps depends on coordinates and $L$, the nonlinear diffusion equation was derived. We found that the diffusion coefficient in this nonlinear equation is directly proportional to the concentration of impurities, as it had been proposed in earliest papers. The derived nonlinear diffusion equation was solved numerically for the case of spherical symmetry.
\end{abstract}

PACS numbers: 66.30.--h

\section{Introduction}

From the theory of Brownian motion it follows that the diffusion velocity and the maximum penetration depth of impurity atoms must be finite. For describing of this physical reality, the following nonlinear diffusion equation was proposed in an ancient paper [1]:

*corresponding author; e-mail: AYanavi@takas.lt 


$$
\begin{aligned}
& j(x, t)=-D_{n} n(x, t) \frac{\mathrm{d}}{\mathrm{d} x} n(x, t), \\
& \frac{\mathrm{d}}{\mathrm{d} t} n(x, t)=D_{n} \frac{\mathrm{d}}{\mathrm{d} x}\left[n(x, t) \frac{\mathrm{d}}{\mathrm{d} x} n(x, t)\right],
\end{aligned}
$$

with the diffusion coefficient directly proportional to the concentration of impurities $n(x, t)$ :

$$
D(x, t)=D_{n} n(x, t) .
$$

Under this assumption, the mass flux of impurities $j$ is proportional to their concentration and to the concentration gradient of impurities, which contradicts the Fick first law. However, this model includes the physically realistic assumption according to which the mass flux (1) $j$ at the point $x+\Delta x$ differs from zero only when the impurity gradient and concentration are different from zero at the point $x$ :

$$
J(x+\Delta x, t) \approx-D_{n} n(x, t) \frac{n(x+\Delta x, t)-n(x, t)}{\Delta x} .
$$

Physically, the latter equation means that the length of the jump of diffusing particles from the point $x$ to $x+\Delta x$ in the diffusion process is not greater than $\Delta x$ and it is possible only when a diffusing particle exists at the point $x$. In this model, the maximum penetration depth of diffusing impurities has finite value, and it grows with finite velocity. We may assume that the jump length of diffusing atoms or molecules from one position to another in fluids or solids has a defined value $L$. In gases, $L$ may be the average free path of the diffusing particles. We assume that the process of spreading of impurities is similar to other diffusion processes and, in the nonlinear case, can be described by the nonlinear diffusion equation [1-4]. In the case of three dimensions, we propose that frequency of the jumps depends upon the particle coordinates, and the time variation of concentration of the impurities $n(x, y, z, t)$ is given by [2-6]:

$$
\begin{aligned}
& \frac{\partial n}{\partial t}=I_{x}+I_{y}+I_{z} \\
& I_{x}=\nu(x+L, y, z) n(x+L, y, z)+\nu(x-L, y, z) n(x-L, y, z)-2 \nu n \\
& I_{y}=\nu(x, y+L, z) n(x, y+L, z)+\nu(x, y-L, z) n(x, y-L, z)-2 \nu n \\
& I_{z}=\nu(x, y, z+L) n(x, y, z+L)+\nu(x, y, z-L) n(x, y, z-L)-2 \nu n
\end{aligned}
$$

Here, $L$ is the average free path of the particles of smoke or molecules of impurities in gases, or the length of the jump of molecules or atoms from the one equilibrium position to another. In fluids and solids, $\nu$ is the frequency of those jumps or collisions. Expanding the functions $n$ and $\nu$ in the Taylor power series and including three main terms in the $n$ and $\nu$ expansions, we obtain the following nonlinear equation: 


$$
\frac{\partial n}{\partial t}=L^{2} \nu \Delta n+2 L^{2}(\operatorname{grad} \nu)(\operatorname{grad} n)+L^{2} n \Delta \nu=L^{2} \Delta(\nu n) .
$$

It should be noted that this equation is different from the equation derived in [4], where the Taylor series expansion of the function $\nu$ included only two main terms, rather than three.

The average frequency $\nu(x, y, z, t)$ of collisions in gas is expressed [5] by

$$
\nu(x, y, z, t)=\sigma N(x, y, z, t) u \sqrt{2} .
$$

Here, $\sigma$ is the collision cross-section of the diffusing particles, $N$ is the density of the solvent particles, $u \sqrt{2}$ is the relative velocity of the diffusing atoms and solvent particles. In the case of diffusion of impurities in the crystals via vacancies, $N$ in Eq. (3) is equal to the density of the vacancies.

Assuming that the coordinate $z$ is fixed, we can use the latter formula in the two-dimensional case. For different processes, the $N$ in (7) can have different meanings. Usually for diffusion processes we have $(n / N) \ll 1$, but we must take into account the fact that concentration of solvent and impurities atoms or molecules during the diffusion process becomes $N+n$ for a non-equilibrium process for diffusion in dense gases or $N-n$ for a diffusion process at equilibrium conditions for diffusion in diluted solvents or in gases of small density. For diffusion in solids through vacancies, the sum of impurity density $n$ and density of free vacancies $N$ must be equal to the maximum density of vacancies $N_{v}$. For the diffusion process in a gas, it means that pressure in the solvent gas $N k T$ and the average pressure in the diffusion region $(N-n) k T+n k T$ must be equal. For this case in the region of a high intensity source we can replace $N$ in (3) by $n$.

In our case, only the collisions between the impurity atoms or molecules and molecules of solvent gas or vacancies in solid state materials or fluids are important. Then, modelling diffusion from the source of the high intensity, we can use the approximation that the number of solvent particles on the diffusion front is equal to $N_{\mathrm{f}} \approx n / 2 \approx N / 2$. In this case we obtain

$$
\nu_{\mathrm{f}}(x, y, z, t)=\frac{1}{2} \sigma n(x, y, z, t) u \sqrt{2} .
$$

Here we assume that a thin region adjacent to the diffusion front with quickly decreasing impurity concentration has a half of the vacancies filled (in a fluid or solid state material), or a half of molecules replaced by impurity molecules (in a gas). Near the source, all molecules of the solvent gas are replaced by molecules of impurities or all vacancies of solid state are filled with the diffusing atoms, and then in this region we have self-diffusion of impurities. Then we have

$$
\nu(x, y, z, t)=\sigma n(x, y, z, t) u \sqrt{2} .
$$

Taking into account that experimental diffusion coefficients are usually defined on the front of diffusion profiles, we can replace $\operatorname{grad} \nu \operatorname{by} \operatorname{grad} \nu_{\mathrm{f}}$ on the right side of Eq. (6). Substituting (8) in (6), we obtain the following nonlinear equation: 


$$
\frac{\partial n}{\partial t}=\frac{D}{N}\left[n \Delta n+(\operatorname{grad} n)^{2}\right]
$$

where the diffusion coefficient $D$ is defined by the equation

$$
D=\sqrt{2} L^{2} \sigma u N .
$$

For diffusion of impurities in solids by vacancies, $N$ means the maximum density of vacancies or impurities. Earlier we proposed that on the diffusion front half of solvent particles of gas are replaced by impurities $N_{\mathrm{f}} \approx n / 2 \approx N / 2$, but in the case of pure solvent or at the region of source $N$ is constant. In this case, the diffusion coefficient can be expressed as follows:

$$
D=2 D_{\mathrm{f}} .
$$

The coefficient $D$ can be compared with the diffusion coefficient $D_{\mathrm{f}}$ for the region of the diffusion front.

Now we can write the nonlinear diffusion equation (10) in the three-dimensional spherical case

$$
\frac{\partial n}{\partial t}=\frac{D}{N}\left[n\left(\frac{\mathrm{d}^{2} n}{\mathrm{~d} r^{2}}+\frac{2}{r} \frac{\mathrm{d} n}{\mathrm{~d} r}\right)+\left(\frac{\mathrm{d} n}{\mathrm{~d} r}\right)^{2}\right] .
$$

The obtained nonlinear diffusion equation in one dimension was proposed in [1] and [7] for calculation of diffusion profiles of impurities in silicon.

\section{Results of the numerical solution of the nonlinear diffusion equation}

The graphs presented below compare solutions of two equations - linear equation

$$
\frac{\partial n}{\partial t}=D \Delta n=D\left(\frac{\mathrm{d}^{2} n}{\mathrm{~d} r^{2}}+\frac{2}{r} \frac{\mathrm{d} n}{\mathrm{~d} r}\right)
$$

and nonlinear equation (13). Parameter values are $D=0.182 \mathrm{~cm}^{2} / \mathrm{s}$ and $N=2.68 \times 10^{19} \mathrm{~cm}^{-3}$. Those values of the diffusion coefficient $D$ and solvent concentration $N$ correspond to diffusion of $\mathrm{CO}$ in air at normal pressure [8]. The radius of the diffusion source is assumed to be equal to $r_{0}=1 \mathrm{~cm}$.

Figures 1-3 have been obtained using the boundary condition

$$
\left.n\right|_{r=r_{0}, t \geq 0}=N=2.68 \times 10^{19} \mathrm{~cm}^{-3}
$$

and the initial condition

$$
\left.n\right|_{r>r_{0}, t=0}=0
$$

(i.e., impurity concentration on the surface of the source is constant and equal to $N$, whereas the initial impurity concentration in the entire region $r>r_{0}$ is equal to zero).

All the curves shown in Figs. 1-3 have been obtained numerically, using the method of lines [9]. However, the solution of the linear equation with the boundary 


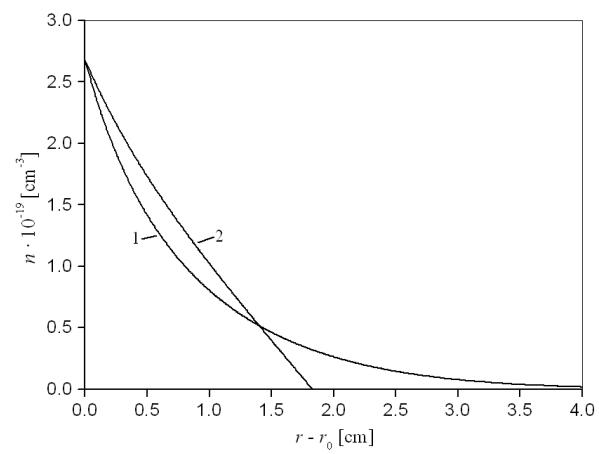

Fig. 1. Solutions of linear and nonlinear diffusion equations at $t=10 \mathrm{~s}$ assuming that impurity concentration on the surface of the impurity source is constant and equal to $2.68 \times 10^{19} \mathrm{~cm}^{-3}$, and the initial impurity concentration in the region $r>r_{0}$ is zero. Curve 1 - linear equation, curve 2 - nonlinear equation.

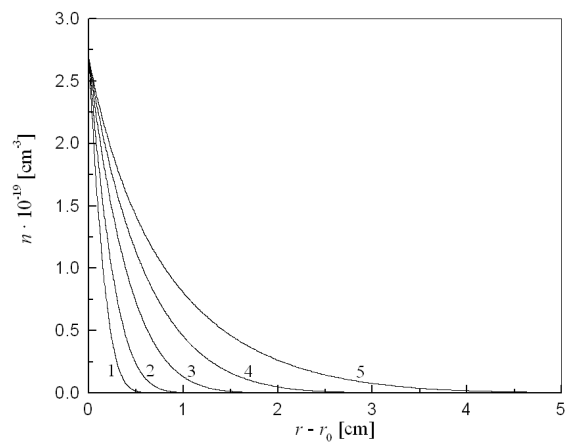

Fig. 2. Solutions of the linear diffusion equation at different moments of time (conditions are the same as in Fig. 1$) .1-t=0.1 \mathrm{~s}, 2-t=0.3 \mathrm{~s}, 3-t=1 \mathrm{~s}, 4-t=3 \mathrm{~s}$, $5-t=10 \mathrm{~s}$.

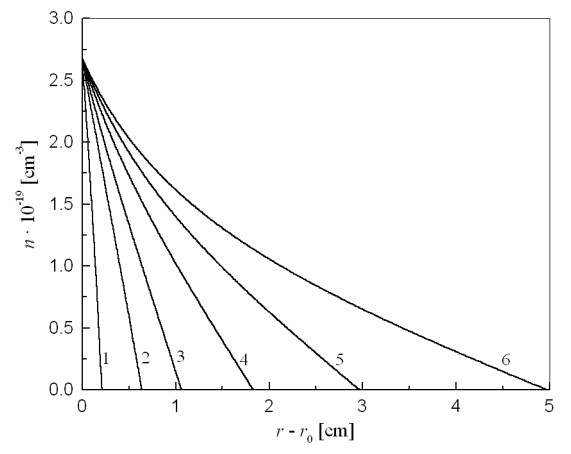

Fig. 3. Solutions of the nonlinear diffusion equation at different moments of time (conditions are the same as in Fig. 1). $1-t=0.1 \mathrm{~s}, 2-t=1 \mathrm{~s}, 3-t=3 \mathrm{~s}, 4-t=10 \mathrm{~s}$, $5-t=30 \mathrm{~s}, 6-t=100 \mathrm{~s}$. 
conditions (15a) and (15b) can also be expressed analytically as follows [6]:

$$
n(r, t)=N \frac{r_{0}}{r} \operatorname{erfc}\left(\frac{r-r_{0}}{2 \sqrt{D t}}\right), \quad r \geq r_{0} .
$$

The curves presented in Fig. 2, as well as curve 1 in Fig. 1, coincide perfectly with those predicted by Eq. (16). It means that numerical errors are negligible.

\section{Discussion}

The results presented above in Figs. 1-3 show that the shape of the solution of the nonlinear diffusion equation is significantly different from the shape of the solution of the linear equation. In the case of the nonlinear equation, the radial profile of impurity distribution has a much smaller curvature than in the case of the linear equation and is close to a linear function, except at longer times $(t>30 \mathrm{~s})$, when the superlinear character of this profile becomes more pronounced. The solution of the nonlinear equation is exactly equal to zero beyond a certain distance from the diffusion source, whereas the solution of the linear equation asymptotically approaches zero. Consequently, the largest differences between the two functions are observed at long distances. Those differences are understandable, because the nonlinear equation has been obtained based on the assumption that the diffusion process can occur only with finite velocity.

Despite the difference in shape, the relative difference between solutions of linear and nonlinear diffusion equations at $t=10 \mathrm{~s}$ and at shorter times does not exceed $20 \%$ up to the point of intersection of the two radial distributions (see Fig. 1). However, those differences become larger at longer times.

\section{References}

[1] A.J. Janavičius, V. Stukaitè, D.J. Zanevičius, Electron Techn. Ser. 2: Semiconductor Dev. 160, 27 (1983) (in Russian).

[2] A.J. Janavičius, Random Processes and Transfer Phenomena, Šiauliai university, Šiauliai 2002, p. 85 (in Lithuanian).

[3] A.J. Janavičius, Phys. Lett. A 224, 159 (1997).

[4] A.J. Janavičius, G. Lūža, D. Jurgaitis, Acta Phys. Pol. A 105, 475 (2004).

[5] B.-F. Apostol, Phys. Lett. A 235, 363 (1997).

[6] M.E. Gliksman, Diffusion in Solids, Wiley, New York 2000, p. 472.

[7] W.-S. Wang, Y.-H. Lo, IEEE Trans. Electron Dev. ED-30, 1828 (1983).

[8] Laboratory Guide of Physics, Ed. L.L. Goldin, Nauka, Moscow 1983, p. 704 (in Russian).

[9] N.K. Madsen, R.F. Sincovec, Trans. Math. Software 5, 326 (1979). 